\section{Wavefront optics and retinal image quality}

\author{
Cristina M. Oliveira, Sandra Franco \\ Centre of Physics, University of Minho, \\ Braga, Portugal
}

\begin{abstract}
With the increasing importance of optical aberrations in both vision research and clinical practice, it becomes necessary for vision scientists to have a thorough understanding of the concepts behind wavefront optics. Therefore, in this review, we provide some basic wave optics concepts useful to understand wavefront analysis, and describe the application of Zernike polynomials in the decomposition of aberrations. A general description of the human eye optical structure is given, followed by a more detailed analysis of the optical components of the eye and optical aberrations, and their secondary effect on overall optical quality. We further provide an overview of the current corneal and ocular wavefront sensing methods.
\end{abstract}

\section{Introduction}

Vision is a complex process encompassing several stages and involving various physiological components, collectively referred to as the visual system.

The human visual system captures information about the environment by detecting light with the eyes. The light entering the eyes is focused onto the retina, where it is converted into a neural signal by photoreceptors in a process designated as transduction. The neural signals are then transported by the connecting pathways (a system of millions of nerves) and integrated into the visual cortex, the part of the brain that processes visual information.

Therefore, visual process starts with the optical system of the eye forming a retinal image. An optically perfect eye would focus all light rays from a point source into a single image point on the retina, producing a perfect image. However, the optical quality of real eyes is affected by three types of optical limitations: aberrations, diffraction, and scattering. In this paper, we will focus on the role of monochromatic aberrations on retinal image quality.

Monochromatic aberrations cause light rays from a point object not to converge to a single retinal point, reducing the quality of the image (Figure 1). Therefore, eyes' optical properties have a crucial role in image and vision quality.

\section{Basic principles of wavefront optics: wavefront aberrations and optical path difference}

There are two interchangeable approaches to define wavefront aberrations: the optical path difference (OPD) and the wavefront shape.

The optical path length (OPL) specifies the number of oscillations performed by a propagating ray of light. A simple formula for computing this optical distance is to multiply the physical path length by the refractive index. The analysis of the behavior of light rays by OPL relies on Fermat's principle of least time, which states that the light from an object point will reach an image point taking the shortest optical path.

In a perfect system, the OPL will be the shortest, and equal, for all light rays traveling from the object point to a unique image point and therefore OPD will be zero. On the other hand, light rays passing through an aberrated system will arrive at the image point with different phases, as they travel along different optical path lengths. Thus, optical aberrations can be described as differences in the OPL.

A wavefront is a conceptual surface that connects all the light rays emerging from a point source with the same temporal phase, i.e. with the same OPL. For example, in the void, parallel light from an object at infinity (or sufficiently distant) will create a plane wavefront. On the other hand, light emerging from or converging to a point will originate a spherical shaped wavefront, with the center of curvature in that point (Figure 2).

Accordingly, in the case of a perfect human eye, all image-forming rays will be in phase, producing a spherical wavefront centered on the image point. In reality, the eye's optical system is not perfect. This means that light rays passing through the pupil arrive with difference phases. These rays produce an aberrated wavefront that deviates from the ideal shape (Figure 3). The departure of this actual wavefront from its ideal spherical shape is the OPD and defines the wavefront aberration (WA).

In ocular optics, WAs (x,y) are usually described mathematically by the infinite Zernike polynomial series. The series is comprised by orthogonal terms defined in the unit circle, meaning that they are mathematically independent of one another. This property is essential for modeling the wavefront shape because it allows each polynomial function of the Zernike expansion to extract individual components of the WA. Each of these components represents a particular type of WA. Hence, Zernike decomposition provides a good description of the eye's optical properties, through
Correspondence: Sandra Franco, Centre of Physics, University of Minho, Campus de Gualtar, 4710-057 Braga, Portugal.

Tel. +35.1253.604067 - Fax: +35.1253.678981.

E-mail: sfranco@fisica.uminho.pt

Key words: wavefront optics, retina, image quality.

Acknowledgments: this work was supported by the European Regional Development Fund (FEDER) through the COMPETE Program and by the Portuguese Foundation for Science and Technology (FCT) in the framework of the Strategic Project PEST-C/FIS/UI607/2011.

Contributions: the authors contributed equally.

Conflict of interests: the authors declare no potential conflict of interests.

Received for publication: 20 November 2012.

Revision received: 3 April 2013.

Accepted for publication: 23 April 2013.

This work is licensed under a Creative Commons Attribution NonCommercial 3.0 License (CC BYNC 3.0).

(C) Copyright C.M. Oliveira and S. Franco., 2013

Licensee PAGEPress, Italy

Optometry Reports 2013; 3:e1

doi:10.4081/optometry.2013.e1

the association of each particular aberration with a Zernike mode Znm, where n defines the order of the term and $m$ is the frequency of the term. The modes of the second radial order $(n=2)$ are collectively known as lower-order aberrations (LOAs) and represent the defocus (including myopia and hyperopia) and astigmatism errors. Higher-order aberrations (HOAs) are represented by Zernike modes of radial order higher than 2 . The contribution of an individual Zernike aberration mode to the actual WA at a given aperture (or pupil size) is given by the magnitude of its corresponding coefficient.

The Optical Society of America (OSA) established a set of recommendations for reporting the Zernike polynomials and a single notation scheme, frequently used in visual optics. ${ }^{1}$ Table 1 presents the Zernike coefficients for different notations, as well as its common name.

\section{Descriptors of optical and retinal image quality}

Besides the Zernike polynomial modeling of the WA described above, several objective descriptors of the optical and image quality can be derived from wavefront information. ${ }^{2,3}$ Opticalquality metrics [e.g. root-mean-square (RMS) 
error] describe the optical properties of the eye and image-quality metrics (e.g. pointspread function, modulation transfer function) and predict the effect of those properties on image optical quality at the fovea. ${ }^{3,4}$ The main difference between these approaches is that the former quantify the wavefront error in the pupil plane, whereas the latter describe the wavefront error in the plane of the retina.

\section{Root mean square}

In some cases, a mean value of the entire wavefront such as the RMS wavefront error, is more convenient than having each wavefront point. The RMS value is a useful quantification of the wavefront variance as a whole or of its individual components, thanks to the orthonormal nature of the Zernike series. Thus, we can compute RMS error for any combination of Zernike terms by applying the following formula:

$$
\mathrm{RMS}^{2}=\sum\left[\mathrm{Z}_{\mathrm{n}}{ }^{\mathrm{m}}\right]^{2}
$$

\section{Point spread function}

The point spread function (PSF) is the image of a point source produced by the eye's optical system (Figure 4). In an optically per- fect eye, a point source produces a retinal image with high contrast and compact form. The retinal image of a point source in an aberrated eye, on the other hand, has lower contrast and is blurred by the ocular optics. A high (almost infinite) variety of PSF images can be obtained according to the nature and magnitude of the eye's optical aberrations.

A popular metric of image quality of the PSF in aberrated eyes is the Strehl ratio. the Strehl ratio is a contrast metric defined as the ratio between the actual maximum intensity of the PSF (in the presence of aberrations) and the maximum intensity for an optically perfect PSF (only diffraction-limited). The Strehl ratio ranges from 0 to 1 , with 1 indicating a diffractionlimited optical system free of aberrations.

\section{Optical transfer function}

Optical transfer function (OTF) metrics are designed to quantify the retinal image quality for sinusoidal gratings. Eye's optical quality can affect the image of a grading by reducing the contrast and shifting the phase. Variations in contrast and phase from the object to the image are called modulation transfer function (MTF) and phase transfer function (PTF), respectively.
Eye's OTF encompasses both the MTF and the PTF; the MTF can be derived by taking the magnitude of the OTF, whereas the PTF can be determined from the OTF's phase. A high-quality OTF is defined by high MTF values and low PTF values.

\section{Optical components of the eye and aberrations sources}

Light entering the eye is sequentially refracted by a series of transparent media with different refractive indices. Of these, the cornea and the crystalline lens are the most important refractive elements. The combination of their optical properties, the pupil size, the retina, and their respective relative positions defines the optical quality of the eye.

Retinal image formation starts when visible light emanating from an object reaches the cornea, where the first refraction of the light rays occurs. Then, the light travels through the aqueous humor, passing through the pupil (a diaphragm controlled by the iris). The aqueous humor further refracts light to reach the crystalline lens. Having passed the lens, light is

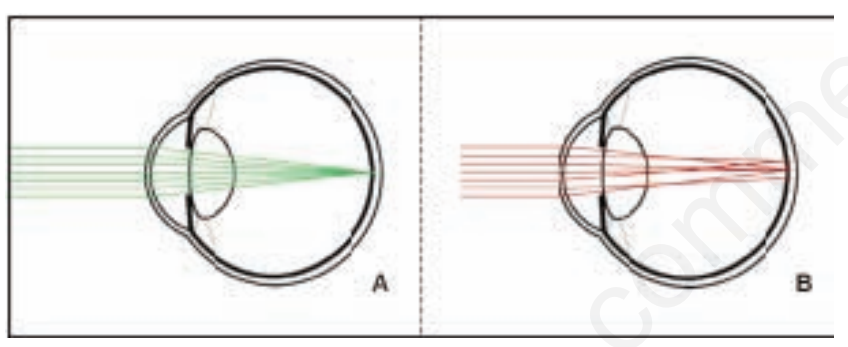

Figure 1. Schematic representation of parallel rays from an object at infinity traveling through (A) a perfect eye and focusing into a single point on the retina; and (B) an aberrated eye and intersecting the retina at different locations, thus forming a blurred image.
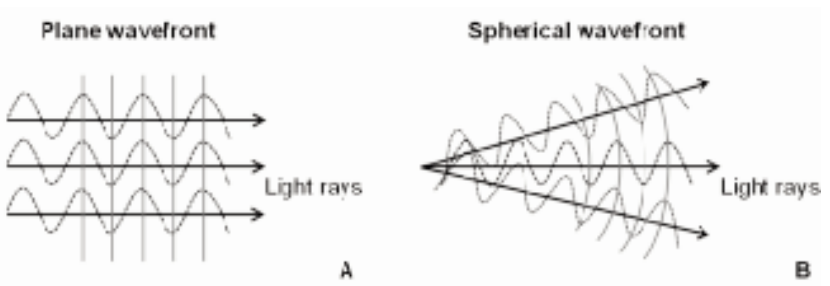

Figure 2. Light rays originating (A) a plane wavefront and (B) a spherical shape wavefront.

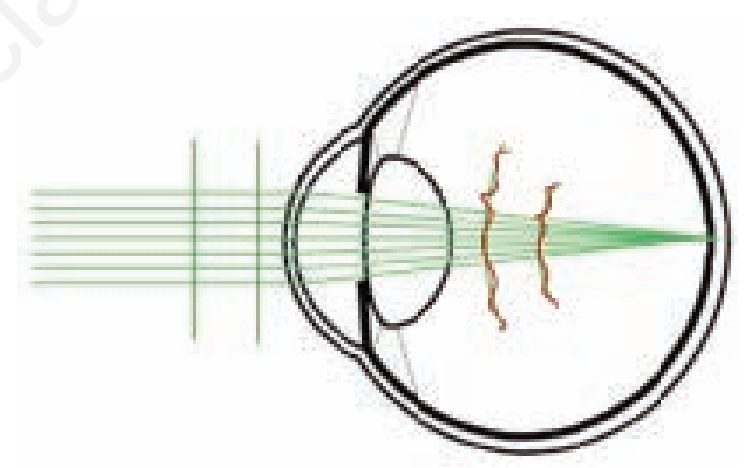

Figure 3. Aberrated wavefront produced by the eye.

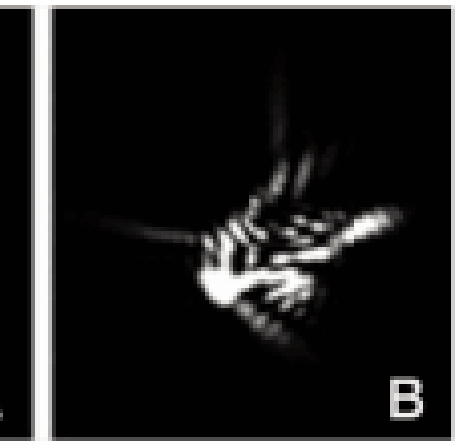

Figure 4. The point spread function for a diffraction-limited eye (A) and a typical aberrated human eye (B).

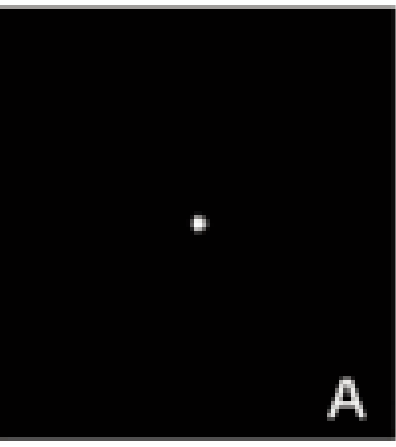


once again refracted, at the lens/vitreous humor interface, forming an image on the retina at the back of the eye.

Accordingly, ocular aberrations are defined by i) surfaces' shape, ii) thickness, iii) the refractive index of the ocular media (tear film, cornea, aqueous, lens, vitreous), and iv) decentering or tilting of the various optical components of the eye with respect to each other. In practice, the anterior corneal surface is the major source of aberrations in normal eyes, followed by the crystalline lens.

\section{Cornea}

The cornea is responsible for two-thirds of the unaccommodated eye's focusing power (when the crystalline lens is relaxed). ${ }^{5}$ Therefore, it has a fundamental role in the overall retinal image quality. The optical properties of this tissue are determined by three aspects: i) its transparency, ii) its refractive index and iii) its surfaces' shapes.

Corneal aberrations mostly arise from its anterior surface. The expressiveness of the anterior corneal surface's optical action is jus-

Table 1. Zernike coefficients notations.

\begin{tabular}{|c|c|c|c|c|c|}
\hline n & m & Name & OSA & Noll & Malacara \\
\hline 0 & 0 & Piston & $\mathrm{ZO}$ & $\mathrm{Z1}$ & $Z_{0}^{0}$ \\
\hline 1 & -1 & Y-tilt & $\mathrm{Z1}$ & $\mathrm{Z3}$ & $\mathrm{Z}_{0}^{1}$ \\
\hline 1 & 1 & X-tilt & $\mathrm{Z} 2$ & $\mathrm{Z} 2$ & $\mathrm{Z}_{1}^{1}$ \\
\hline 2 & -2 & 450-astig & Z3 & $\mathrm{Z} 5$ & $\mathrm{Z}_{0}^{2}$ \\
\hline 2 & 0 & Defocus & $\mathrm{Z4}$ & $\mathrm{Z} 4$ & $\mathrm{Z}_{1}^{2}$ \\
\hline 2 & 2 & 00-astig & $\mathrm{Z} 5$ & $\mathrm{Z} 6$ & $\mathrm{Z}_{2}^{2}$ \\
\hline 3 & -3 & Y-trefoil & $\mathrm{Z} 6$ & $\mathrm{Z9}$ & $\mathrm{Z}_{0}^{3}$ \\
\hline 3 & -1 & Vertical coma & $\mathrm{Z7}$ & $\mathrm{Z7}$ & $\mathrm{Z}_{1}^{3}$ \\
\hline 3 & 1 & Horizontal coma & $\mathrm{Z8}$ & $\mathrm{Z} 8$ & $\mathrm{Z}_{2}^{3}$ \\
\hline 3 & 3 & X-trefoil & $\mathrm{Z9}$ & $\mathrm{Z10}$ & $\mathrm{Z}_{3}^{3}$ \\
\hline 4 & -4 & Y-tetrafoil & $\mathrm{Z10}$ & $\mathrm{Z15}$ & $\mathrm{Z}_{0}^{4}$ \\
\hline 4 & -2 & Secondary y-astig & Z11 & $\mathrm{Z} 13$ & $\mathrm{Z}_{1}^{4}$ \\
\hline 4 & 0 & Spherical abeeration & $\mathrm{Z12}$ & Z11 & $\mathrm{Z}_{2}^{4}$ \\
\hline 4 & 2 & Secondary x-astig & $\mathrm{Z} 13$ & $\mathrm{Z12}$ & $Z_{3}^{4}$ \\
\hline 4 & 4 & X-tetrafoil & $\mathrm{Z14}$ & $\mathrm{Z14}$ & $\mathrm{Z}_{4}^{4}$ \\
\hline 5 & -5 & Y-pentafoil & $\mathrm{Z} 15$ & Z21 & $\mathrm{Z}_{0}^{5}$ \\
\hline 5 & -3 & Secondary y-trefoil & Z16 & Z19 & $\mathrm{Z}_{1}^{5}$ \\
\hline 5 & -1 & Secondary V-coma & $\mathrm{Z17}$ & $\mathrm{Z17}$ & $\mathrm{Z}_{2}^{5}$ \\
\hline 5 & 1 & Secondary H-coma & $\mathrm{Z18}$ & $\mathrm{Z} 16$ & $\mathrm{Z}_{3}^{5}$ \\
\hline 5 & 3 & Secondary $x$-trefoil & $\mathrm{Z19}$ & $\mathrm{Z18}$ & $\mathrm{Z}_{4}^{5}$ \\
\hline 5 & 5 & X-pentafoil & Z20 & $\mathrm{Z} 20$ & $\mathrm{Z}_{5}^{5}$ \\
\hline
\end{tabular}

$\mathrm{n}$, order of the term; m, frequency of the term; OSA, Optical Society of America.

Table 2. Technical comparison of some commercial available aberrometers.

\begin{tabular}{|c|c|c|c|c|}
\hline \multirow[t]{2}{*}{ Details } & \multicolumn{4}{|c|}{ Device } \\
\hline & OPD-scan & VFA & Allegretto & L80 wave+ \\
\hline Manufacturer & Nidek & Tracey technology & Alcon & Visionix Luneau \\
\hline Method & Automated retinoscopy & Laser ray tracing & Tscherning & Hartmann-Sack \\
\hline Wavelength (nm) & 808 & 650 & 660 & 780 \\
\hline Measurement speed & $>0.4 \mathrm{~s}$ & $>50 \mathrm{~ms}$ & $40 \mathrm{~ms}$ & $0.2 \mathrm{~s}$ \\
\hline Number of sample & 1440 & 256 & 168 & 1500 \\
\hline Pupil size & $6 \mathrm{~mm}$ & $\begin{array}{l}\text { Automatic/user } \\
\text { defined }\end{array}$ & $\begin{array}{c}\text { Automatic/user } \\
\text { defined }\end{array}$ & $3 \mathrm{~mm} / 5 \mathrm{~mm} / 7 \mathrm{~mm}$ \\
\hline Automatic averaging & 3 measures & No & User defined & User defined \\
\hline Accommodation control & Fogging & Fogging & Fogging & Object at infinity \\
\hline $\begin{array}{l}\text { Number of Zernike } \\
\text { polynomials }\end{array}$ & $\begin{array}{c}27 \\
\left(6^{\text {th }} \text { order }\right)\end{array}$ & $\begin{array}{c}27 \\
\left(6^{\text {th }} \text { order }\right)\end{array}$ & $\begin{array}{c}27 \\
\left(6^{\text {th }} \text { order }\right)\end{array}$ & $\begin{array}{c}27 \\
\left(6^{\text {th }} \text { order }\right)\end{array}$ \\
\hline
\end{tabular}

OPD, optical path difference; VFA, visual function analyzer.

tified by its highly curved surface and the considerable difference in refractive index at the interface between the air and itself. The posterior corneal surface has a modest contribution to the total corneal aberrations of normal eyes by virtue of the small difference in refractive index between cornea and aqueous humor, compared to the change at the air-cornea interface. ${ }^{6}$ Accordingly, subtle imperfections in cornea's shape cause light rays to be improperly focused on the retina, resulting in optical aberrations, ${ }^{7}$ changes in the corneal shape due to pathological conditions or refractive surgery/therapy may impact its optical quality and, consequently, the eye's optical performance..$^{8-12}$

\section{Pupil}

The pupil regulates the aperture of the eye's image system, controlling the amount of light falling onto the retina. Larger pupil sizes allow the light rays to enter the eye through cornea and crystalline lens periphery with larger incident angles compared to paraxial rays, increasing the wavefront aberrations. Therefore, the pupil diameter affects the eye's optical quality by magnifying the aberrations with an increasing pupil size. ${ }^{13,14}$ This aberrometric magnification induces a decrease in visual performance. ${ }^{15}$ Moreover, the pupil is not centered with respect to the rest of the eye's components, and its centration changes with changes in the pupil size (including pupil dilatation), illumination and accommodation. ${ }^{16,17}$ Pupil decentration degrade the retinal image quality by increasing monochromatic aberrations, particularly for larger pupil sizes. ${ }^{18,19}$

\section{Crystalline lens}

In its relaxed state during distance vision, the crystalline lens provides one-third of the overall refractive power of the eye. The optical properties of the lens are determined by the surface curvatures, thickness, the refractive index differences at the aqueous-lens and lens-vitreous interfaces and the refractive index gradient distribution within the lens. ${ }^{5}$ The crystalline lens position on the eye, usually decentred and tilted with respect to the cornea, ${ }^{20}$ also affects its optical properties and, consequently, the quality of the eye.

Furthermore, this structure is responsible for increasing the optical power of the eye during accommodation. Changes in lens shape, thickness and refractive index distribution increase its optical power (i.e. reduce its focal length), enabling to focus objects at a wide range of distances. ${ }^{21}$

\section{Retina}

The fovea - a small area of the retina where resolution is maximized - is not centered with the optical axis of the eye. In fact, the optical axis joining the center of curvature of the eye's 
optical structures cannot be defined, due to the decentrations and tilts between ocular surfaces, pupil and fovea. On the other hand, the visual axis can be defined as the line joining the fixation target and the fovea, passing through the nodal points. Early studies predicted that the angle between the optical axis and the visual axis, known as the angle alpha, affect overall eye aberrations. ${ }^{22}$ However, Marcos et al. ${ }^{23}$ found no correlation between the amount of aberrations and the magnitude of the angle alpha.

\section{Measurement of the wavefront aberration of the eye}

\section{Ocular wavefront sensing methods}

Several wavefront sensing techniques have been applied to measure the wavefront aberrations of the human eye. ${ }^{24-27}$ Currently, the most commonly used aberrometers employ laser ray tracing technique, Hartmann-Shack wavefront sensor, Tscherning sensor, and automatic retinoscopy. Table 2 presents some commercially available devices and their characteristic features, either inherent to the technique employed or related with the particular setup of the device.

The techniques employed by the wavefront sensors can be based on serial or parallel principles; serial methods acquire the data points with a one-by-one measurement, whereas parallel methods collect all data points simultaneously. These systems can use single or double-pass principles, according to the number of times the measurement beam passes through the aberrated eye optics. Finally, their measurements can be based on forward projection (i.e. the focal shifts are projected on the retina), or backward projection (i.e. the focal shifts are projected directly on a camera).

\section{Automated retinoscopy}

The automated retinoscopy is based on an objective, serial, and double-pass method. The retina is scanned with a slit light beam and the light reflected back is captured by an array of rotating photodetectors over a $360^{\circ}$ area. The difference in direction and time between the incident beam and the reflected light are used to determine the aberrations along the analyzed meridian. ${ }^{28}$

\section{Hartmann-Shack aberrometry}

The Hartmann-Shack (HS) sensor is the most commonly used wavefront technique. Hartmann-Shack aberrometers employ an objective double-pass method based on backward projection to measure the WA. The data is collected using parallel measurements.

The HS device uses a narrow laser beam that is projected along the line of sight into the eye and is reflected on the retina. As the reflected light comes out of the eye, it collects all the aberrations from the ocular system. Then, the aberrated light passes through a set of relay lenses that projects the pupil plane onto a microlens array. This microlens array consisting of several lenslets, all with the same diameter and focal length, splits up the light into many individual beams and focuses multiple spots in its focal plane. The spot array pattern is recorded by a charged coupled device (CCD) camera placed in the focal plane of the lenslet array. ${ }^{25}$ Displacements of each retinal spot with respect to the reference (ideal) position are determined in order to reconstruct the wavefront aberration in the entire pupil at once. The focal shift of each spot is measured to obtain information about the aberrations present in the ocular system.

\section{Laser ray-tracing}

The laser ray-tracing technique consists of a light beam sequentially projected by a scanning mirror through different locations in the eye's pupil onto the retina. Each spot pattern on the retina is imaged by simple optics and captured by a CCD. The ocular wavefront is reconstructed from the sequence of the image displacements as compared to the reference spots. Therefore, ray tracing aberrometry is based on a serial, double-pass method using forward projection, which can be implemented either subjectively or objectively. ${ }^{29}$

\section{Tscherning aberrometry}

Tscherning aberrometry employs a parallel, double-pass method using forward projection and can be implemented either subjectively or objectively. In the Tscherning wavefront sensor, a dot pattern mask (regular holes) is used to project several laser beams onto the retina. The ocular aberrations in the eye cause the rays from different parts of the pupil to focus at different positions on the retina. Therefore, the ocular wavefront aberrations can be computed from the entire spot array pattern captured at once by an ophthalmoscope. ${ }^{30}$

\section{Corneal wavefront aberration measurements}

The shape (elevation) data provided by corneal topography can be used to determine the corneal wavefront aberrations.

Modern corneal topography techniques are able to provide shape information of the entire cornea, instead of simply measuring its center. Such complete information can be leveraged to compute a wavefront aberration function that describes the optical properties of the cornea. Currently, the most frequently used topographers are based on Placido-disk, slit-scanning, and Scheimpflug technologies. Placido disk systems directly measure the curvature of the anterior corneal surface by analyzing a specularly reflected image of rings on the cornea. $0 n$ the other hand, slit-scanning and Scheimpflug imaging enable the assessment of both anterior and posterior corneal surfaces, through projection of the light from a slit onto the cornea. Several optical sections of the eye are acquired and used to reconstruct a 3-dimensional model of the cornea. In particular, due to the implementation of the Scheimpflug principle, these systems provide multiple sharp images from the anterior corneal surface to the posterior crystalline capsule..$^{31,32}$

In an optically perfect eye composed by single surface system, namely a perfect cornea, all light rays coming from a point in infinity will reach the focal point at the same phase, that is, with the same OPL. Consequently, their OPD is null. However, in reality, the presence of corneal aberrations implies that marginal light rays arrive at the focal point in different phases relative to the axial ray, thus forming an aberrated wavefront. Therefore, corneal wavefront aberration can be computed as the difference in OPL between the axial and marginal rays. This approach can be employed to determine both anterior surface and total corneal aberrations, using corneal shape data provided by elevation-based topography. ${ }^{31}$ The subtraction of anterior surface aberration from the total corneal aberration allows to compute posterior corneal surface aberrations.

Corneal aberrations can also be computed by considering corneal shape as the actual wavefront, since its aberrations arise from its shape, and determine the difference between this surface and an ideal aberration free wavefront. This ideal wavefront is generally defined by fitting the corneal elevation data to a Cartesian oval surface aligned with the sighting center. The OPD is obtained by multiplying the residual surface profile by the refractive index difference between air and cornea. This method has been applied to determine both anterior and posterior corneal surfaces aberrations, and total corneal aberrations as the sum of these two ${ }^{33}$ However, total corneal aberrations cannot be accurately determined as the sum of these two, since the wavefront incident on the posterior corneal surface is already deformed by the refraction of the anterior surface. ${ }^{34,35}$

\section{Crystalline lens wavefront aberrations}

The study of the optical properties of in vivo crystalline lens is a difficult task, due to its position behind the cornea and pupil, and posterior surface's distortions resulting from the lens refractive index gradient. Hence, the optical properties of the lens have been indirectly measured by combining the analyses of corneal and ocular aberrations. Artal et al.$^{36}$ com- 
pared internal optics aberrations indirectly measured following the subtraction method with internal aberrations directly measured after neutralizing anterior corneal aberrations (using water-filled goggles). The authors found no significant difference between directly and indirectly measured values of internal optics aberrations, thus validating the indirect method.

\section{Conclusions}

In conclusion, this review provides some basic wave optics concepts which help understand wavefront analysis in optometric practice, and describe the application of Zernike polynomials in the decomposition of aberrations. A general description of the human eye optical structure is described with a detailed analysis of the eye components optical aberrations and their secondary effect on overall optical quality. Wavefront analysis is a powerful tool in eye care practice in several disciplines (e.g. refractive surgery), and increases knowledge on contact lenses and quality of vision assessment.

\section{References}

1. Thibos L, Applegate RA, Schwiegerling JT, Webb R. Standards for reporting the optical aberrations of eyes. J Refract Surg 2000;18:S652-60.

2. Atchison DA, Scott DH, Cox MJ. Mathematical treatment of ocular aberrations : a user's guide. OSA Trends Opt Photo 2000;35:110-30.

3. Cheng X, Thibos LN, Bradley A. Estimating visual quality from wavefront aberration measurements. J Refract Surg 2003;19: 579-84.

4. Marsack JD, Thibos LN, Applegate RA. Metrics of optical quality derived from wave aberrations predict visual performance. J Vision 2004;4:322-8.

5. Atchison DA, Smith G. Optics of the human eye. Oxford, UK: ButterworthHeinemann; 2000.

6. Barbero S, Marcos S, Merayo-Lloves J. Corneal and total optical aberrations in a unilateral aphakic patient. J Cataract Refr Surg 2002;28:1594-600.

7. Maloney RK, Bogan SJ, Waring GO 3rd. Determination of corneal image-forming properties from corneal topography. Am J Ophthalmol 1993;115:31-41.

8. Barbero S, Marcos S, Merayo-Lloves J, Moreno-Barriuso E. Validation of the esti- mation of corneal aberrations from videokeratography in keratoconus. J Refract Surg 2002;18:263-70.

9. Oliver KM, Hemenger RP, Corbett MC, et al. Corneal Optical aberrations induced by photorefractive keratectomy. J Refract Surg 1997;13:246-54.

10. Marcos S, Barbero S, Llorente L, MerayoLloves J. Optical response to LASIK surgery for myopia from total and corneal aberration measurements. Investigative 0phth Vis Sci 2001;42:3349-56.

11. Buzzonetti L, Iarossi G, Valente P, et al. Comparison of wavefront aberration changes in the anterior corneal surface after laser-assisted subepithelial keratectomy and laser in situ keratomileusis: preliminary study. J Cataract Refr Surg 2004;30:1929-33.

12. Hiraoka T, Matsumoto Y, Okamoto F, et al. Corneal higher-order aberrations induced by overnight orthokeratology. Am J Ophthal 2005;139:429-36.

13. Wang Y, Zhao K, Jin Y, et al. Changes of higher order aberration with various pupil sizes in the myopic eye. J Refract Surg 2003;19(Suppl. 2):S270-4.

14. Yang Y, Wu F. Technical note: comparison of the wavefront aberrations between natural and pharmacological pupil dilations. Ophthal Physl 0pt 2007;27:220-3.

15. Applegate RA, Hilmantel G, Howland HC, et al. Corneal first surface optical aberrations and visual performance. J Refract Surg 2000;16:507-14.

16. Walsh G. The effect of mydriasis on the pupillary centration of the human eye. Ophthal Physl Opt 2007;8:178-82.

17. Wilson MA, Campbell MC, Simonet P, et al. Change of pupil centration with change of illumination and pupil size. Optometry Vision Sci 1992;69:129-36.

18. Walsh G, Charman WN. The effect of pupil centration and diameter on ocular performance. Vision Res 1988;28:659-65.

19. Artal P, Marcos S, Iglesias I, Green DG. Optical modulation transfer and contrast sensitivity with decentered small pupils in the human eye. Vision Res 1996;36:357586.

20. Dunne MC, Elawad MA, Barnes DA. Determination of the influence of effectivity upon residual astigmatism. Acta Ophthalmol 1997;75:170-3.

21. Dubbelman M, Van der Heijde GL, Weeber HA, Vrensen GF. Changes in the internal structure of the human crystalline lens with age and accommodation. Vision Res 2003;43:2363-75.

22. Meeteren A Van, Dunnewold CJW. Image quality of the human eye for eccentric entrance pupils. Vision Res 1983;23:573-9.

23. Marcos S, Burns SA, Prieto PM, et al. Investigating sources of variability of monochromatic and transverse chromatic aberrations across eyes. Vision Res 2001;41: 3861-71.

24. Walsh G, Charman WN, Howland HC. Objective technique for the determination of monochromatic aberrations of the human eye. J Opt Soc Am A 1984;1:987-92.

25. Liang J, Grimm B, Goelz S, Bille JF. Objective measurement of wave aberrations of the human eye with the use of a Hartmann-Shack wave-front sensor. J Opt Soc Am A 1994;11:1949-57.

26. Mierdel P, Krinke HE, Wiegand W, et al. [Measuring device for determining monochromatic aberration of the human eye]. [Article in German]. Ophthalmologe 1997;94:441-5.

27. Iglesias I, Berrio E, Artal P. Estimates of the ocular wave aberration from pairs of double-pass retinal images. J 0pt Soc Am A 1998;15:2466-76.

28. MacRae S, Fujieda M. Slit skiascopic-guided ablation using the Nidek laser. J Refract Surg 2000;16:576-80.

29. Navarro R, Moreno-Barriuso E. Laser raytracing method for optical testing. Optics Lett 1999;24:951-3.

30. Mrochen M, Kaemmerer M, Mierdel P, et al. Principles of Tscherning aberrometry. J Refract Surg 2000;16:570-1.

31. Braaf B, Dubbelman M, Van der Heijde RG, Sicam VA. Performance in specular reflection and slit-imaging corneal topography. Optometry Vision Sci 2009;86:467-75.

32. Guirao A, Artal P. Corneal wave aberration from videokeratography: accuracy and limitations of the procedure. J Opt Soc Am A 2000;17:955-65.

33. Chen M, Yoon G. Posterior corneal aberrations and their compensation effects on anterior corneal aberrations in keratoconic eyes. Investigative Ophth Vis Sci 2008;49:5645-52.

34. Dubbelman M, Sicam VA, Van der Heijde RGL. The contribution of the posterior surface to the coma aberration of the human cornea. J Vision 2007;7:1-8.

35. Nakagawa T, Maeda N, Kosaki R, et al. Higher-order aberrations due to the posterior corneal surface in patients with keratoconus. Investigative Ophth Vis Sci 2009;50:2660-5.

36. Artal P, Guirao a, Berrio E, Williams DR. Compensation of corneal aberrations by the internal optics in the human eye. $\mathrm{J}$ Vision 2001;1:1-8. 